\title{
Inclusion Properties of Certain Subclasses of $p$-Valent Functions Associated with the Integral Operator
}

\author{
Tamer M. Seoudy \\ Department of Mathematics, Faculty of Science, Fayoum University, Fayoum 63514, Egypt \\ Correspondence should be addressed to Tamer M. Seoudy; tms00@fayoum.edu.eg \\ Received 2 May 2014; Accepted 18 May 2014; Published 29 May 2014 \\ Academic Editor: Ming-Sheng Liu
}

Copyright (C) 2014 Tamer M. Seoudy. This is an open access article distributed under the Creative Commons Attribution License, which permits unrestricted use, distribution, and reproduction in any medium, provided the original work is properly cited.

The purpose of the present paper is to introduce two subclasses of $p$-valent functions by using the integral operator and to investigate various properties for these subclasses.

\section{Introduction}

Let $\mathscr{A}(p)$ denote the class of functions of the following form:

$$
f(z)=z^{p}+\sum_{j=1}^{\infty} a_{p+j} z^{p+j}, \quad(p \in \mathbb{N}=\{1,2,3, \ldots\}),
$$

which are analytic and $p$-valent in the open unit disc $\mathbb{U}=\{z \in$ $\mathbb{C}:|z|<1\}$. Let $\mathscr{P}_{k}(p, \gamma)$ be the class of functions $g$ analytic in $\mathbb{U}$ satisfying $g(0)=p$ and

$$
\int_{0}^{2 \pi}\left|\frac{\Re\{g(z)\}-\gamma}{p-\gamma}\right| d \theta \leq k \pi, \quad\left(z=r e^{i \theta} ; k \geq 2 ; 0 \leq \gamma<p\right) .
$$

The class $\mathscr{P}_{k}(p, \gamma)$ was introduced by Aouf [1] and we note the following:

(i) the class $\mathscr{P}_{k}(1, \gamma)=\mathscr{P}_{k}(\gamma)$ was introduced by Padmanabhan and Parvatham [2];

(ii) the class $\mathscr{P}_{k}(1,0)=\mathscr{P}_{k}$ was introduced by Pinchuk [3];

(iii) $\mathscr{P}_{2}(p, \gamma)=\mathscr{P}(p, \gamma)$ is the class of functions with positive real part greater than $\gamma(0 \leq \gamma<p)$;

(iv) $\mathscr{P}_{2}(1, \gamma)=\mathscr{P}(\gamma)$ is the class of functions with positive real part greater than $\gamma(0 \leq \gamma<1)$;

(v) $\mathscr{P}_{2}(1,0)=\mathscr{P}$ is the class of functions with positive real part.
From (1), we have $g \in \mathscr{P}_{k}(p, \gamma)$ if and only if there exists $g_{1}, g_{2} \in \mathscr{P}(p, \gamma)$ such that

$$
g(z)=\left(\frac{k}{4}+\frac{1}{2}\right) g_{1}(z)-\left(\frac{k}{4}-\frac{1}{2}\right) g_{2}(z), \quad(z \in \mathbb{U}) .
$$

It is known that [4] the class $\mathscr{P}_{k}(\gamma)$ is a convex set.

Motivated essentially by Jung et al. [5], Liu and Owa [6] introduced the integral operator $Q_{\beta, p}^{\alpha}: \mathscr{A}(p) \rightarrow \mathscr{A}(p)(\alpha \geq$ $0 ; \beta>-p ; p \in \mathbb{N})$ as follows:

$Q_{\beta, p}^{\alpha} f(z)$

$$
= \begin{cases}\left(\begin{array}{c}
p+\alpha+\beta-1 \\
p+\beta-1
\end{array}\right) \frac{\alpha}{z^{\beta}} \int_{0}^{z}\left(1-\frac{t}{z}\right)^{\alpha-1} t^{\beta-1} f(t) d t & (\alpha>0), \\
f(z) & (\alpha=0) .\end{cases}
$$

For $f \in \mathscr{A}(p)$ given by (1) and then from (4), we deduce that

$$
\begin{array}{r}
Q_{\beta, p}^{\alpha} f(z)=z^{p}+\frac{\Gamma(\alpha+\beta+p)}{\Gamma(\beta+p)} \sum_{j=1}^{\infty} \frac{\Gamma(\beta+p+j)}{\Gamma(\alpha+\beta+p+j)} a_{p+j} z^{p+j} \\
(\alpha \geq 0 ; \beta>-p) .
\end{array}
$$

It is easily verified from (5) that (see [6])

$$
z\left(Q_{\beta, p}^{\alpha+1} f(z)\right)^{\prime}=(\alpha+\beta+p) Q_{\beta, p}^{\alpha} f(z)-(\alpha+\beta) Q_{\beta, p}^{\alpha} f(z) .
$$


We note that (i) the one-parameter family of integral operator $Q_{\beta, 1}^{\alpha}=Q_{\beta}^{\alpha}$ was defined by Jung et al. [5] and studied by Aouf [7] and Gao et al. [8].

(ii) Consider

$$
Q_{c, p}^{1} f(z)=F_{c, p}(f)(z)=\frac{c+p}{z^{c}} \int t^{c-1} f(z) d t, \quad(c>-p),
$$

where the operator $F_{c, p}$ is the generalized Bernardi-LiberaLivingston integral operator (see [9]).

We have the following known subclasses $\mathcal{S}_{k}(p, \gamma)$ and $\mathscr{C}_{k}(p, \gamma)$ of the class $\mathscr{A}(p)$ for $0 \leq \gamma, \eta<p$, and $k \geq 2$ which are defined by

$$
\begin{gathered}
\mathcal{S}_{k}(p, \gamma)=\left\{f \in \mathscr{A}(p): \frac{z f^{\prime}(z)}{f(z)} \in \mathscr{P}_{k}(p, \gamma), z \in \mathbb{U}\right\}, \\
\mathscr{C}_{k}(p, \gamma)=\left\{f \in \mathscr{A}(p): \frac{\left(z f^{\prime}(z)\right)^{\prime}}{f^{\prime}(z)} \in \mathscr{P}_{k}(p, \gamma), z \in \mathbb{U}\right\} .
\end{gathered}
$$

Next, by using the integral operator $Q_{\beta, p}^{\alpha}$, we introduce the following classes of analytic functions for $0 \leq \gamma<p$ and $k \geq 2$ :

$$
\begin{aligned}
& \mathcal{S}_{k}(p, \alpha ; \gamma)=\left\{f \in \mathscr{A}(p): Q_{\beta, p}^{\alpha} f(z) \in \mathcal{S}_{k}(p, \gamma)\right\}, \\
& \mathscr{C}_{k}(p, \alpha ; \gamma)=\left\{f \in \mathscr{A}(p): Q_{\beta, p}^{\alpha} f(z) \in \mathscr{C}_{k}(p, \gamma)\right\} .
\end{aligned}
$$

We also note that

$$
f \in \mathscr{C}_{k}(p, \alpha ; \gamma) \Longleftrightarrow \frac{z f^{\prime}}{p} \in \mathcal{S}_{k}(p, \alpha ; \gamma) .
$$

In particular, we set $\mathcal{S}_{k}(1, \alpha ; \gamma)=\mathcal{S}_{k}(\alpha ; \gamma)$ and $\mathscr{C}_{k}(1, \alpha ; \gamma)=$ $\mathscr{C}_{k}(\alpha ; \gamma)$.

The following lemma will be required in our investigation.

Lemma 1 (see [10]). Let $u=u_{1}+i u_{2}$ and $v=v_{1}+i v_{2}$ and let $\Psi(u, v)$ be a complex-valued function satisfying the following conditions:

(i) $\Psi(u, v)$ is continuous in a domain $D \in \mathbb{C}^{2}$;

(ii) $(0,1) \in D$ and $\Psi(1,0)>0$;

(iii) $\mathfrak{R}\left\{\Psi\left(i u_{2}, v_{1}\right)\right\}>0$ whenever $\left(i u_{2}, v_{1}\right) \in D$ and $v_{1} \leq$ $-(1 / 2)\left(1+u_{2}^{2}\right)$.

If $h(z)=1+c_{1} z+c_{2} z^{2}+\cdots$ is analytic in $\mathbb{U}$ such that $\left(h(z), z h^{\prime}(z)\right) \in D$ and $\mathfrak{R}\left\{\Psi\left(h(z), z h^{\prime}(z)\right)\right\}>0$ for $z \in \mathbb{U}$, then $\mathfrak{R}\left\{\Psi\left(h(z), z h^{\prime}(z)\right)\right\}>0$ in $\mathbb{U}$.

Lemma 2 (see [11]). Let $p(z)$ be analytic in $\mathbb{U}$ with $p(0)=a$ and $\mathfrak{R}\{p(z)\}>0, z \in \mathbb{U}$. Then, for $s>0$ and $\mu \in \mathbb{C} \backslash\{-1\}$,

$$
\mathfrak{R}\left\{p(z)+\frac{s z p^{\prime}(z)}{p(z)+\mu}\right\}>0, \quad\left(|z|<r_{0}\right),
$$

where $r_{0}$ is given by

$$
r_{0}=\frac{|\mu+1|}{\sqrt{A+\left(A^{2}-\left|\mu^{2}-1\right|\right)^{1 / 2}}}, \quad A=2(s+1)^{2}+|\mu|^{2}-1
$$

and this radius is the best possible.

Lemma 3 (see [12]). Let $\psi$ be convex and let $g$ be starlike in $\mathbb{U}$. Then, for $F$ analytic in $\mathbb{U}$ with $F(0)=1,((\psi * F g) /(\psi * g))$ is contained in the convex hull of $F(\mathbb{U})$.

In this paper, we obtain several inclusion properties of the classes $\mathcal{S}_{k}(p, \alpha ; \gamma)$ and $\mathscr{C}_{k}(p, \alpha ; \gamma)$ associated with the operator $Q_{\beta, p}^{\alpha}$.

\section{Main Results}

Unless otherwise mentioned, we assume throughout this paper that $k \geq 2, \alpha \geq 0, \beta>0,0 \leq \gamma<p$, and $p \in \mathbb{N}$.

Theorem 4. One has

$$
\mathcal{S}_{k}(p, \alpha+1 ; \gamma) \subset \mathcal{S}_{k}(p, \alpha ; \gamma)
$$

Proof. We begin by setting

$$
\begin{aligned}
\frac{z\left(Q_{\beta, p}^{\alpha+1} f(z)\right)^{\prime}}{Q_{\beta, p}^{\alpha+1} f(z)}= & (p-\gamma) h(z)+\gamma \\
= & \left(\frac{k}{4}+\frac{1}{2}\right)\left\{(p-\gamma) h_{1}(z)+\gamma\right\} \\
& -\left(\frac{k}{4}-\frac{1}{2}\right)\left\{(p-\gamma) h_{2}(z)+\gamma\right\},
\end{aligned}
$$

where $h_{i}$ is analytic in $\mathbb{U}$ with $h_{i}(0)=1, i=1,2$. Using the identity (6) in (14) and differentiating the resulting equation with respect to $z$, we obtain

$$
\begin{aligned}
& \frac{z\left(Q_{\beta, p}^{\alpha} f(z)\right)^{\prime}}{Q_{\beta, p}^{\alpha} f(z)}=\{\gamma+(p-\gamma) h(z) \\
& \left.\quad+\frac{(p-\gamma) z h^{\prime}(z)}{(p-\gamma) h(z)+\gamma+\alpha+\beta}\right\} \in \mathscr{P}_{k}(p, \gamma) .
\end{aligned}
$$

This implies that

$$
h_{i}(z)+\frac{z h_{i}^{\prime}(z)}{(p-\gamma) h_{i}(z)+\gamma+\alpha+\beta} \in \mathscr{P}, \quad(z \in \mathbb{U} ; i=1,2) .
$$

We form the functional $\Psi(u, v)$ by choosing $u=h_{i}(z)$ and $v=z h_{i}^{\prime}(z)$ :

$$
\Psi(u, v)=u+\frac{v}{(p-\gamma) u+\gamma+\alpha+\beta} .
$$


Clearly, the first two conditions of Lemma 1 are satisfied. Now, we verify condition (iii) as follows:

$$
\begin{aligned}
\mathfrak{R}\left\{\Psi\left(i u_{2}, v_{1}\right)\right\} & =\mathfrak{R}\left\{\frac{v_{1}}{(p-\gamma) i u_{2}+\gamma+\alpha+\beta}\right\} \\
& \leq-\frac{(\gamma+\alpha+\beta)\left(1+u_{2}^{2}\right)}{2\left[(p-\gamma)^{2} u_{2}^{2}+(\gamma+\alpha+\beta)^{2}\right]}<0 .
\end{aligned}
$$

Therefore applying Lemma $1, h_{i} \in \mathscr{P}(i=1,2)$ and consequently $h \in \mathscr{P}_{k}$ for $z \in \mathbb{U}$. This completes the proof of Theorem 4.

Theorem 5. One has

$$
\mathscr{C}_{k}(p, \alpha+1 ; \gamma) \subset \mathscr{C}_{k}(p, \alpha ; \gamma) \text {. }
$$

Proof. Applying (10) and Theorem 4, we observe that

$$
\begin{aligned}
f & \in \mathscr{C}_{k}(p, \alpha+1 ; \gamma) \\
& \Longleftrightarrow \frac{z f^{\prime}}{p} \in \mathcal{S}_{k}(p, \alpha+1 ; \gamma) \Longrightarrow \frac{z f^{\prime}}{p} \in \mathcal{S}_{k}(p, \alpha ; \gamma) \\
& \Longleftrightarrow f \in \mathscr{C}_{k}(p, \alpha ; \gamma),
\end{aligned}
$$

which evidently proves Theorem 5 .

Theorem 6. If $f \in \mathcal{S}_{k}(p, \alpha ; \gamma)$, then $F_{c, p}(f) \in \mathcal{S}_{k}(p, \alpha$; $\gamma)(c \geq 0)$, where the generalized Libera integral operator $F_{c, p}$ is defined by (7).

Proof. Let $f \in \mathcal{S}_{k}(p, \alpha ; \gamma)$ and set

$$
\begin{aligned}
\frac{z\left(Q_{\beta, p}^{\alpha} F_{c, p}(f)(z)\right)^{\prime}}{Q_{\beta, p}^{\alpha} F_{c, p}(f)(z)}= & (p-\gamma) h(z)+\gamma \\
= & \left(\frac{k}{4}+\frac{1}{2}\right)\left\{(p-\gamma) h_{1}(z)+\gamma\right\} \\
& -\left(\frac{k}{4}-\frac{1}{2}\right)\left\{(p-\gamma) h_{2}(z)+\gamma\right\},
\end{aligned}
$$

where $h$ is analytic in $\mathbb{U}$ with $h(0)=1$. From (21), we have

$$
z\left(Q_{\beta, p}^{\alpha} F_{c, p}(f)(z)\right)^{\prime}=(c+p) Q_{\beta, p}^{\alpha} f(z)-c Q_{\beta, p}^{\alpha} F_{c, p}(f)(z) \text {. }
$$

Then, by using (21) and (22), we obtain

$$
(c+p) \frac{Q_{\beta, p}^{\alpha} f(z)}{Q_{\beta, p}^{\alpha} F_{c, p}(f)(z)}=(p-\gamma) h(z)+\gamma+c .
$$

Taking the logarithmic differentiation on both sides of (23) with respect to $z$ and multiplying by $z$, we have

$$
\begin{aligned}
& \frac{1}{p-\gamma}\left(\frac{z\left(Q_{\beta, p}^{\alpha} f(z)\right)^{\prime}}{Q_{\beta, p}^{\alpha} f(z)}-\gamma\right) \\
& =h(z)+\frac{z h^{\prime}(z)}{(p-\gamma) h(z)+\gamma+c} \in \mathscr{P}_{k} .
\end{aligned}
$$

This implies that

$$
\left\{h_{i}(z)+\frac{z h_{i}^{\prime}(z)}{(p-\gamma) h_{i}(z)+\gamma+c}\right\} \in \mathscr{P}, \quad(z \in \mathbb{U} ; i=1,2) .
$$

We form the functional $\Psi(u, v)$ by choosing $u=h_{i}(z)$ and $v=z h_{i}^{\prime}(z)$ :

$$
\Psi(u, v)=u+\frac{v}{(p-\gamma) u+\gamma+c} .
$$

Then clearly $\Psi(u, v)$ satisfies all the properties of Lemma 1. Hence, $h_{i} \in \mathscr{P}(i=1,2)$ and consequently $h \in \mathscr{P}_{k}$ for $z \in$ $\mathbb{U}$, which implies that $F_{c, p}(f) \in \mathcal{S}_{k}(p, \alpha ; \gamma)$.

Next, we derive an inclusion property for the subclass $\mathscr{C}_{k}(\alpha ; \gamma)$ involving $F_{c, p}(f)$, which is given by the following theorem.

Theorem 7. If $f \in \mathscr{C}_{k}(p, \alpha ; \gamma)$, then $F_{c, p}(f) \in \mathscr{C}_{k}(p, \alpha$; $\gamma)(c \geq 0)$, where $F_{c, p}$ is defined by (7).

Proof. By applying Theorem 6, it follows that

$$
\begin{aligned}
f \in \mathscr{C}_{k}(p, \alpha ; \gamma) & \Longleftrightarrow \frac{z f^{\prime}}{p} \in \mathcal{S}_{k}(p, \alpha ; \gamma) \\
& \Longrightarrow F_{c, p}\left(\frac{z f^{\prime}}{p}\right) \in \mathcal{S}_{k}(p, \alpha ; \gamma)
\end{aligned}
$$

(by Theorem 5)

$$
\begin{aligned}
& \Longleftrightarrow \frac{z\left(F_{c, p}(f)\right)^{\prime}}{p} \in \mathcal{S}_{k}(p, \alpha ; \gamma) \\
& \Longleftrightarrow F_{c, p}(f) \in \mathscr{C}_{k}(p, \alpha ; \gamma),
\end{aligned}
$$

which proves Theorem 7 .

Theorem 8. If $f \in \mathscr{C}_{k}(p, \alpha+1 ; \gamma)$, for $z \in \mathbb{U}$, then $f \in$ $\mathscr{C}_{k}(p, \alpha ; \gamma)$ for

$$
|z|<r_{0}=\frac{|\mu+1|}{\sqrt{A+\left(A^{2}-\left|\mu^{2}-1\right|\right)^{1 / 2}}}
$$

where $A=2(s+1)^{2}+|\mu|^{2}-1$, with $\mu=((\gamma+\alpha+\beta) /(p-\gamma)) \neq-1$ and $s=(1 /(p-\gamma))$. This radius is the best possible.

Proof. Let $f \in \mathscr{C}_{k}(p, \alpha+1 ; \gamma)$ for $z \in \mathbb{U}$ and let

$$
\begin{aligned}
\frac{z\left(Q_{\beta, p}^{\alpha+1} f(z)\right)^{\prime}}{Q_{\beta, p}^{\alpha+1} f(z)}= & (p-\gamma) h(z)+\gamma \\
= & \left(\frac{k}{4}+\frac{1}{2}\right)\left\{(p-\gamma) h_{1}(z)+\gamma\right\} \\
& -\left(\frac{k}{4}-\frac{1}{2}\right)\left\{(p-\gamma) h_{2}(z)+\gamma\right\},
\end{aligned}
$$


where $h_{i}$ is analytic in $\mathbb{U}$ with $h_{i}(0)=1$ and $\mathfrak{R}\left\{h_{i}(z)\right\}>0$ for $i=1,2$. Using the identity (6) in (29) and differentiating the resulting equation with respect to $z$, we obtain

$$
\begin{aligned}
\frac{1}{p-\gamma}\left\{\frac{z\left(Q_{\beta, p}^{\alpha} f(z)\right)^{\prime}}{Q_{\beta, p}^{\alpha} f(z)}-\gamma\right\} & \\
= & h(z)+\frac{(1 /(p-\gamma)) z h^{\prime}(z)}{h(z)+((\gamma+\alpha+\beta) /(p-\gamma))} \\
= & \left(\frac{k}{4}+\frac{1}{2}\right)\left\{h_{1}(z)+\frac{(1 /(p-\gamma)) z h_{1}^{\prime}(z)}{h_{1}(z)+((\gamma+\alpha+\beta) /(p-\gamma))}\right\} \\
& -\left(\frac{k}{4}-\frac{1}{2}\right)\left\{h_{2}(z)\right. \\
& \left.+\frac{(1 /(p-\gamma)) z h_{2}^{\prime}(z)}{h_{2}(z)+((\gamma+\alpha+\beta) /(p-\gamma))}\right\},
\end{aligned}
$$

where $\mathfrak{R}\left\{h_{i}(z)\right\}>0$ for $i=1,2$. Applying Lemma 2 with $s=$ $((\gamma+\alpha+\beta) /(p-\gamma))$ and $\mu=((\gamma+\alpha+\beta) /(p-\gamma)) \neq-1$, we get

$$
\begin{array}{r}
\mathfrak{R}\left\{h_{i}(z)+\frac{(1 /(p-\gamma)) z h_{i}^{\prime}(z)}{h_{i}(z)+((\gamma+\alpha+\beta) /(p-\gamma))}\right\}>0 \\
\text { for }|z|<r_{0},
\end{array}
$$

where $r_{0}$ is given by (28). This completes the proof of Theorem 8 .

Theorem 9. Let $\phi$ be a convex function and $f \in \mathcal{S}_{2}(\alpha ; \gamma)$. Then $G \in \mathcal{S}_{2}(\alpha ; \gamma)$, where $G=\phi * f$.

Proof. Let $=\phi * f$. Then

$$
Q_{\beta, p}^{\alpha} G(z)=Q_{\beta, p}^{\alpha}(\phi * f)(z)=\phi(z) * Q_{\beta, p}^{\alpha} f(z) .
$$

Also, $f \in \mathcal{S}_{2}(\alpha ; \gamma)$. Therefore, $Q_{\beta, p}^{\alpha} f \in \mathcal{S}_{2}(\gamma)$. By logarithmic differentiation of (32) and after some simplification, we obtain

$$
\frac{z\left(Q_{\beta, p}^{\alpha} G(z)\right)^{\prime}}{p Q_{\beta, p}^{\alpha} G(z)}=\frac{\phi(z) * F(z) Q_{\beta, p}^{\alpha} f(z)}{\phi(z) * Q_{\beta, p}^{\alpha} f(z)},
$$

where $F=z\left(Q_{\beta, p}^{\alpha} f(z)\right)^{\prime} / p Q_{\beta, p}^{\alpha} f(z)$ is analytic in $\mathbb{U}$ and $F(0)=1$. From Lemma 3, we can see that $z\left(Q_{\beta, p}^{\alpha} G(z)\right)^{\prime} /$ $p Q_{\beta, p}^{\alpha} G(z)$ is contained in the convex hull of $F(\mathbb{U})$. Since $z\left(Q_{\beta, p}^{\alpha} G(z)\right)^{\prime} / p Q_{\beta, p}^{\alpha} G(z)$ is analytic in $\mathbb{U}$ and

$$
F(\mathbb{U})=\Omega=\left\{w: \frac{z\left(Q_{\beta, p}^{\alpha} w(z)\right)^{\prime}}{p Q_{\beta, p}^{\alpha} w(z)} \in \mathscr{P}(\gamma)\right\},
$$

then $z\left(Q_{\beta, p}^{\alpha} G(z)\right)^{\prime} / p Q_{\beta, p}^{\alpha} G(z)$ lies in $\Omega$; this implies that $G=$ $\phi * f \in \mathcal{S}_{2}(\alpha ; \gamma)$.

Remark 10. Putting $p=1$ in the above results, we obtain corresponding results for the operator $Q_{\beta}^{\alpha}$.

\section{Conflict of Interests}

The author declares that there is no conflict of interests regarding the publication of this paper.

\section{References}

[1] M. K. Aouf, "A generalization of functions with real part bounded in the mean on the unit disc," Mathematica Japonica, vol. 33, no. 2, pp. 175-182, 1988.

[2] K. S. Padmanabhan and R. Parvatham, "Properties of a class of functions with bounded boundary rotation," Annales Polonici Mathematici, vol. 31, no. 3, pp. 311-323, 1975.

[3] B. Pinchuk, "Functions of bounded boundary rotation," Israel Journal of Mathematics, vol. 10, pp. 7-16, 1971.

[4] K. I. Noor, "On subclasses of close-to-convex functions of higher order," International Journal of Mathematics and Mathematical Sciences, vol. 15, no. 2, pp. 279-290, 1992.

[5] I. B. Jung, Y. C. Kim, and H. M. Srivastava, "The Hardy space of analytic functions associated with certain one-parameter families of integral operators," Journal of Mathematical Analysis and Applications, vol. 176, no. 1, pp. 138-147, 1993.

[6] J.-L. Liu and S. Owa, "Properties of certain integral operator," International Journal of Mathematical Sciences, vol. 3, no. 1, pp. 69-75, 2004.

[7] M. K. Aouf, "Inequalities involving certain integral operators," Journal of Mathematical Inequalities, vol. 2, no. 2, pp. 537-547, 2008.

[8] C.-Y. Gao, S.-M. Yuan, and H. M. Srivastava, "Some functional inequalities and inclusion relationships associated with certain families of integral operators," Computers \& Mathematics with Applications, vol. 49, no. 11-12, pp. 1787-1795, 2005.

[9] J. H. Choi, M. Saigo, and H. M. Srivastava, "Some inclusion properties of a certain family of integral operators," Journal of Mathematical Analysis and Applications, vol. 276, no. 1, pp. 432$445,2002$.

[10] S. S. Miller and P. T. Mocanu, "Second-order differential inequalities in the complex plane," Journal of Mathematical Analysis and Applications, vol. 65, no. 2, pp. 289-305, 1978.

[11] S. Ruscheweyh and V. Singh, "On certain extremal problems for functions with positive real part," Proceedings of the American Mathematical Society, vol. 61, no. 2, pp. 329-334, 1976.

[12] St. Ruscheweyh and T. Sheil-Small, "Hadamard products of Schlicht functions and the Pólya-Schoenberg conjecture," Commentarii Mathematici Helvetici, vol. 48, pp. 119-135, 1973. 


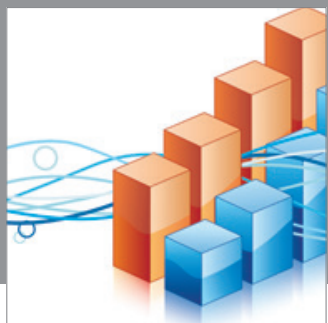

Advances in

Operations Research

mansans

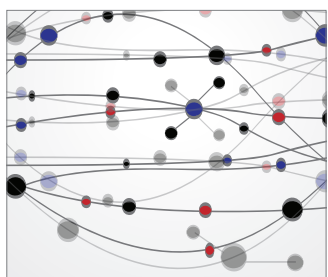

The Scientific World Journal
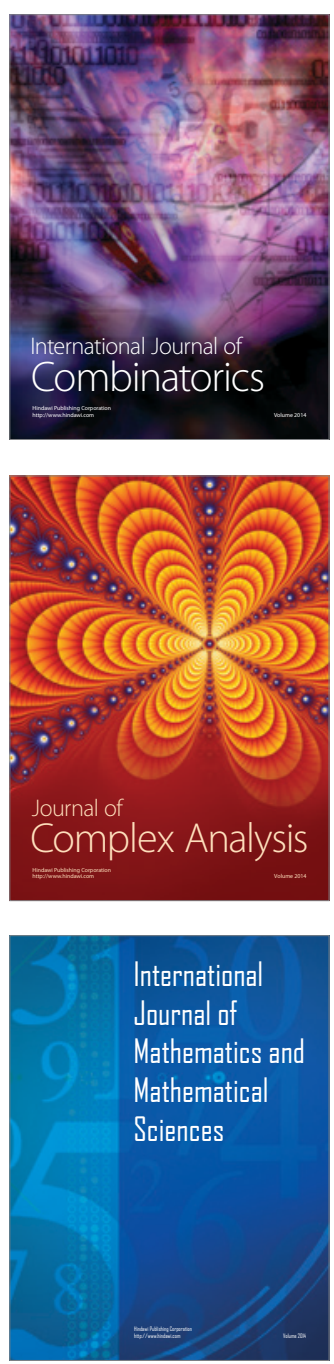
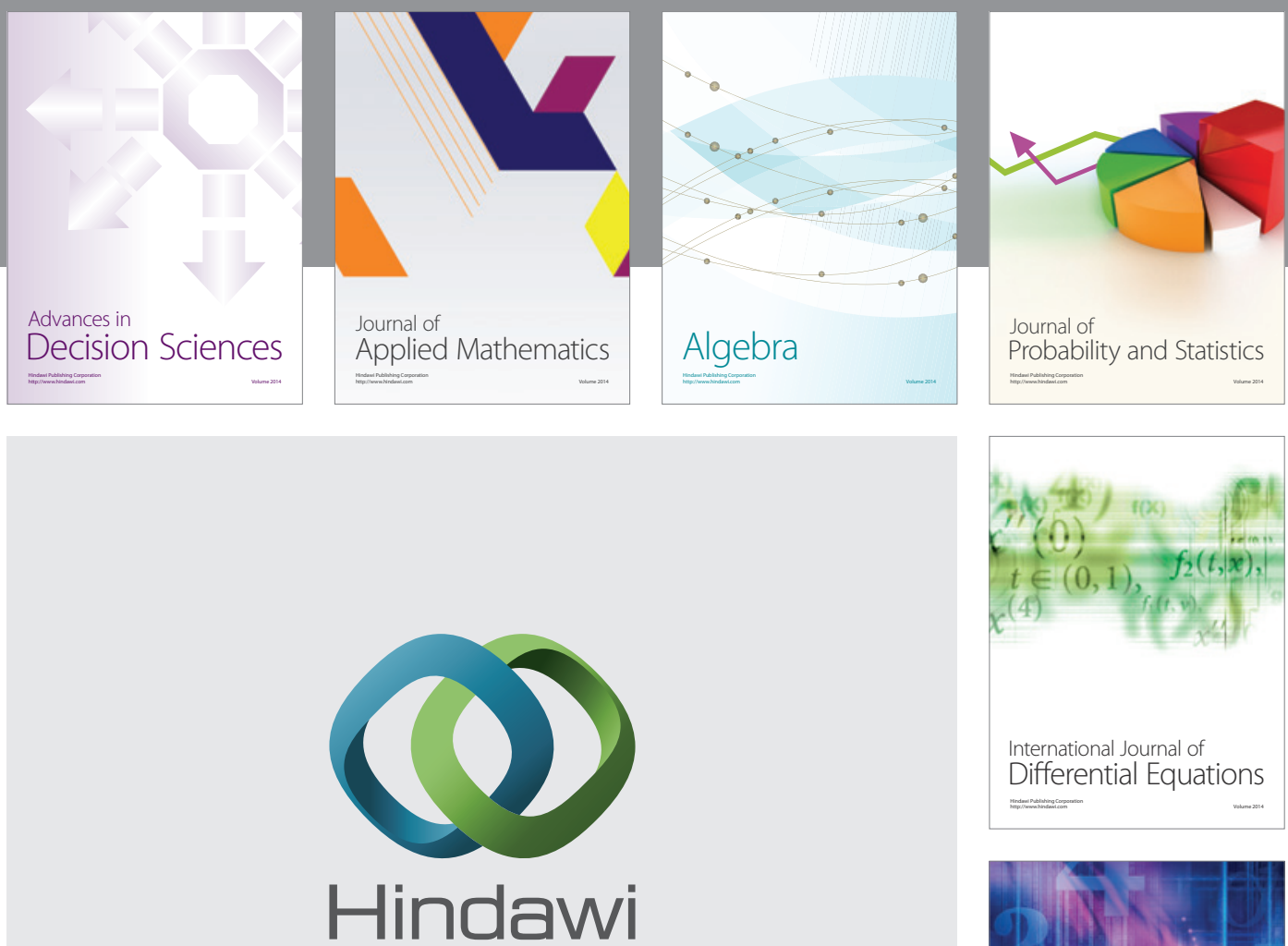

Submit your manuscripts at http://www.hindawi.com
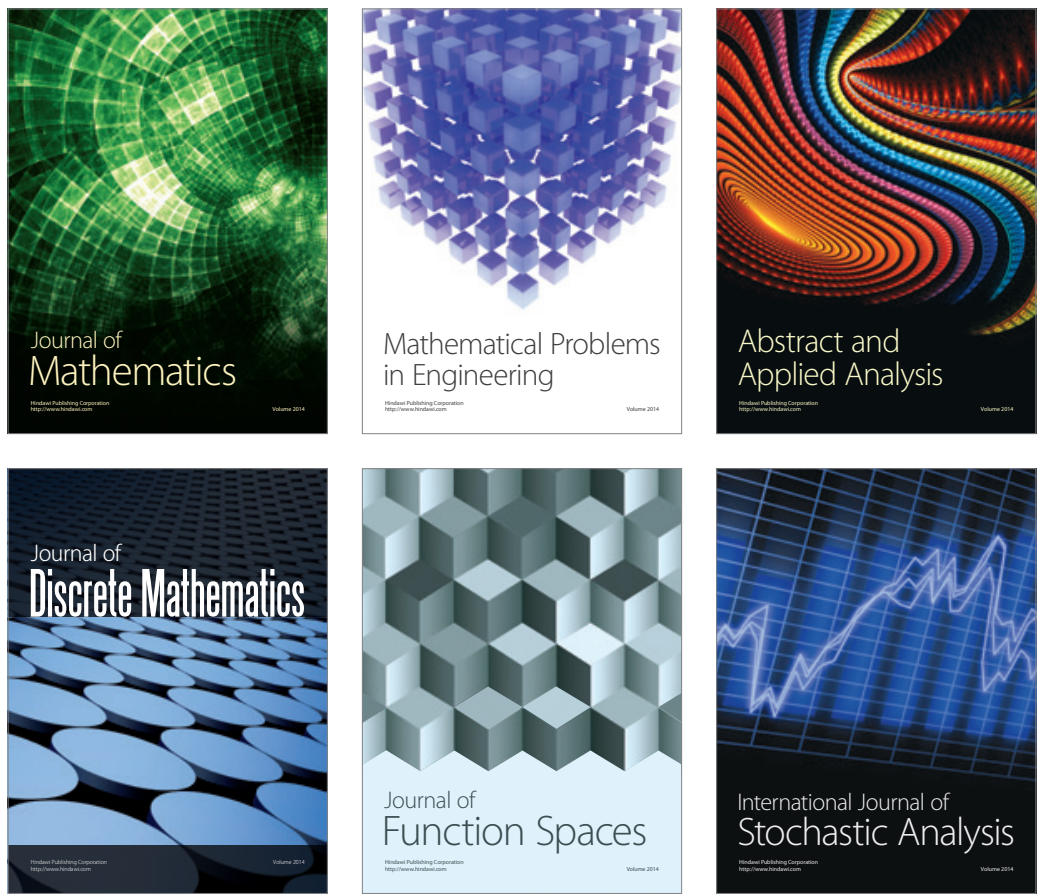

Journal of

Function Spaces

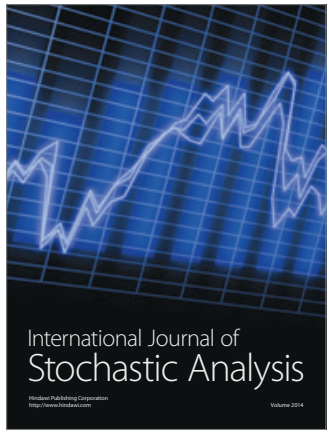

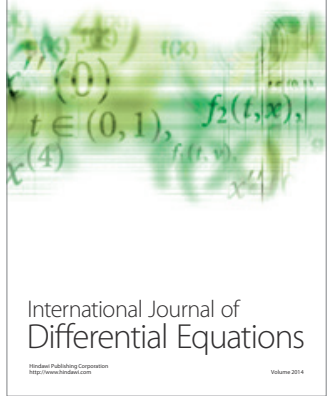
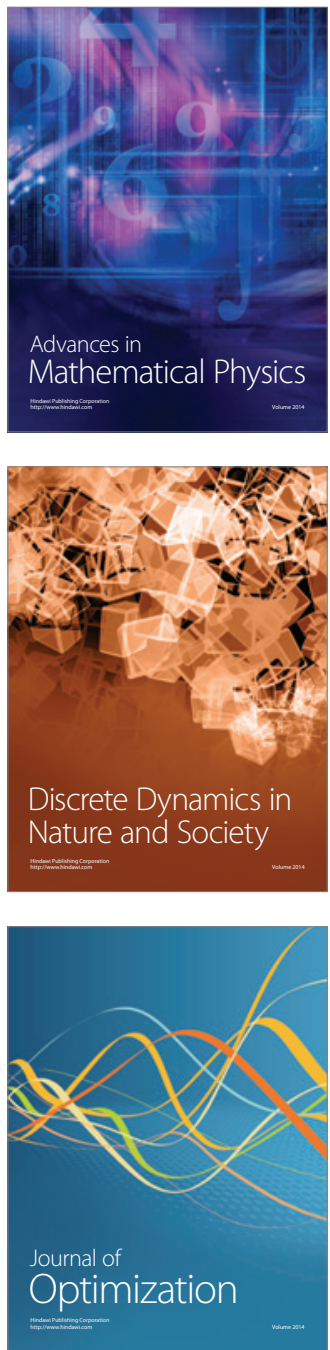